\title{
A secreted decoy of InR antagonizes insulin/IGF signaling to restrict body growth in Drosophila
}

\author{
Naoki Okamoto, Rinna Nakamori, Tomoka Murai, Yuki Yamauchi, Aya Masuda, \\ and Takashi Nishimura ${ }^{1}$ \\ Laboratory for Growth Control Signaling, RIKEN Center for Developmental Biology (CDB), Chuo-ku, Hyogo, $466-8550$ Japan
}

\begin{abstract}
Members of the insulin peptide family have conserved roles in the regulation of growth and metabolism in a wide variety of metazoans. Drosophila insulin-like peptides (Dilps) promote tissue growth through the single insulin-like receptor (InR). Despite the important role of Dilps in nutrient-dependent growth control, the molecular mechanism that regulates the activity of circulating Dilps is not well understood. Here, we report the function of a novel secreted decoy of InR (SDR) as a negative regulator of insulin signaling. SDR is predominantly expressed in glia and is secreted into the hemolymph. Larvae lacking SDR grow at a faster rate, thereby increasing adult body size. Conversely, overexpression of SDR reduces body growth non-cell-autonomously. SDR is structurally similar to the extracellular domain of InR and interacts with several Dilps in vitro independent of Imp-L2, the ortholog of the mammalian insulin-like growth factorbinding protein 7 (IGFBP7). We further demonstrate that SDR is constantly secreted into the hemolymph independent of nutritional status and is essential for adjusting insulin signaling under adverse food conditions. We propose that Drosophila uses a secreted decoy to fine-tune systemic growth against fluctuations of circulating insulin levels.
\end{abstract}

[Keywords: decoy receptor; insulin; insulin/IGF signaling; growth; size; Drosophila]

Supplemental material is available for this article.

Received August 27, 2012; revised version accepted November 26, 2012.

The insulin/insulin-like growth factor (IGF) signaling (IIS) pathway is an evolutionarily conserved endocrine signaling pathway that controls a wide variety of processes, including growth and development (Nakae et al. 2001; Edgar 2006; Hietakangas and Cohen 2009). The central players in this pathway are insulin-like peptides, which include insulin, IGF-1, and IGF-II in mammals (Nakae et al. 2001); 40 insulinlike peptides in worms (Pierce et al. 2001; WormBase, http:// www.wormbase.org); and the seven canonical Drosophila insulin-like peptides (Dilps) in flies that can promote body growth (Brogiolo et al. 2001; Ikeya et al. 2002). These secreted ligands transmit intercellular signals through the activation of insulin receptor tyrosine kinase (insulin-like receptor [InR] in Drosophila), leading to the activation of the PI3-kinase (PI3K) signaling pathway.

In mammals, six classic IGF-binding proteins (IGFBPs) bind to IGF-I and IGF-II with high affinity in serum and modulate IGF activity (Thissen et al. 1994). Less than $5 \%$ of the IGFs in the circulation are free, and most IGFs are bound in the complex, which consists of IGF-I or IGF-II, IGFBP3, and an acid-labile subunit (ALS). This complex is believed to be the principle carrier form of IGFs. These

${ }^{1}$ Corresponding author

E-mail t-nishimura@cdb.riken.jp

Article is online at http://www.genesdev.org/cgi/doi/10.1101/gad.204479.112. proteins either enhance or dampen the IIS pathway by extending the half-life of IGFs, by altering the local and systemic availability of IGFs, or by preventing them from binding to the receptor (Hwa et al. 1999). In addition, an IGFBP-related protein, IGFBP7, binds to IGFs with comparatively low affinity and has been demonstrated to be a potent tumor suppressor in a wide variety of cancers. Insects also express an IGFBP-like protein, referred to as Imp-L2, that resembles IGFBP7 (Sloth Andersen et al. 2000). Imp-L2 binds to Dilp-2 and Dilp-5 and acts as a non-cellautonomous inhibitor of IIS during development (Honegger et al. 2008; Alic et al. 2011). However, it remains unknown whether other factors besides Imp-L2 regulate the seven Dilps in the extracellular space.

Here, we characterize a secreted decoy of InR (SDR) that binds to Dilps and antagonizes IIS during development. Our biochemical and genetic analyses suggest that SDR belongs to a novel class of functional insulin-binding proteins and that it acts in a manner complementary to Imp-L2 in Drosophila.

Results

SDR negatively regulates body growth

To shed light on the regulatory mechanisms of body growth during development, we conducted an RNAi screen of 
putative secreted proteins containing a predicted signal peptide ( $\mathrm{N}$ Okomoto and $\mathrm{T}$ Nishimura, unpubl.). We found that the ubiquitous knockdown of CG3837 by Tub-Gal4 increased larval size, resulting in larger adult flies (Fig. 1A). CG3837 shows extensive sequence similarity to the extracellular domain of InR (Fig. 1B; Supplemental Fig. S1). Pursuant to our functional analysis, we named this gene secreted decoy of InR (SDR).

To investigate the loss-of-function phenotype of $S D R$, we generated $S D R$ mutants by imprecise excision of a $P$-element insertion. We obtained a deletion line, $S D R^{1}$, and a precise excision line, $S D R^{\text {cont }}$ (hereafter referred to as the control), which we used as a genotypically matched control (Fig. 1C; Supplemental Fig. S2). SDR ${ }^{1}$ lacks the predicted transcriptional start site, the $5^{\prime}$ untranslated region, and the $\mathrm{N}$-terminal 310 amino acids of the coding region, including the signal peptide. We also generated a null allele, $S D R^{D f}$, by deleting the entire coding region using an FRT-mediated interchromosomal recombination technique. Two independent SDR deletion mutants were fully viable and fertile when homozygous but exhibited increased adult body weight in both males and females (Fig. 1D). We observed similar results in a transheterozygote of the $S D R^{1}$ allele with the $S D R^{D f}$ allele, suggesting that $S D R^{1}$ is a genetic null (Fig. 1D; Supplemental Fig. S3A).

Increases in IIS promote larval body size by increasing the larval growth rate (Edgar 2006; Hietakangas and Cohen 2009). SDR was continuously expressed throughout the larval period (Fig. 1E). A detailed time-course analysis of body weight revealed that $S D R$ mutants grew more rapidly at all larval stages (Fig. $1 \mathrm{~F}$ ). The size of $S D R$ mutant eggs and newly hatched first instar larvae were identical to the control (Fig. 1G). However, a difference was already detectable at the early second instar and became more pronounced at the third instar (Fig. 1F). The
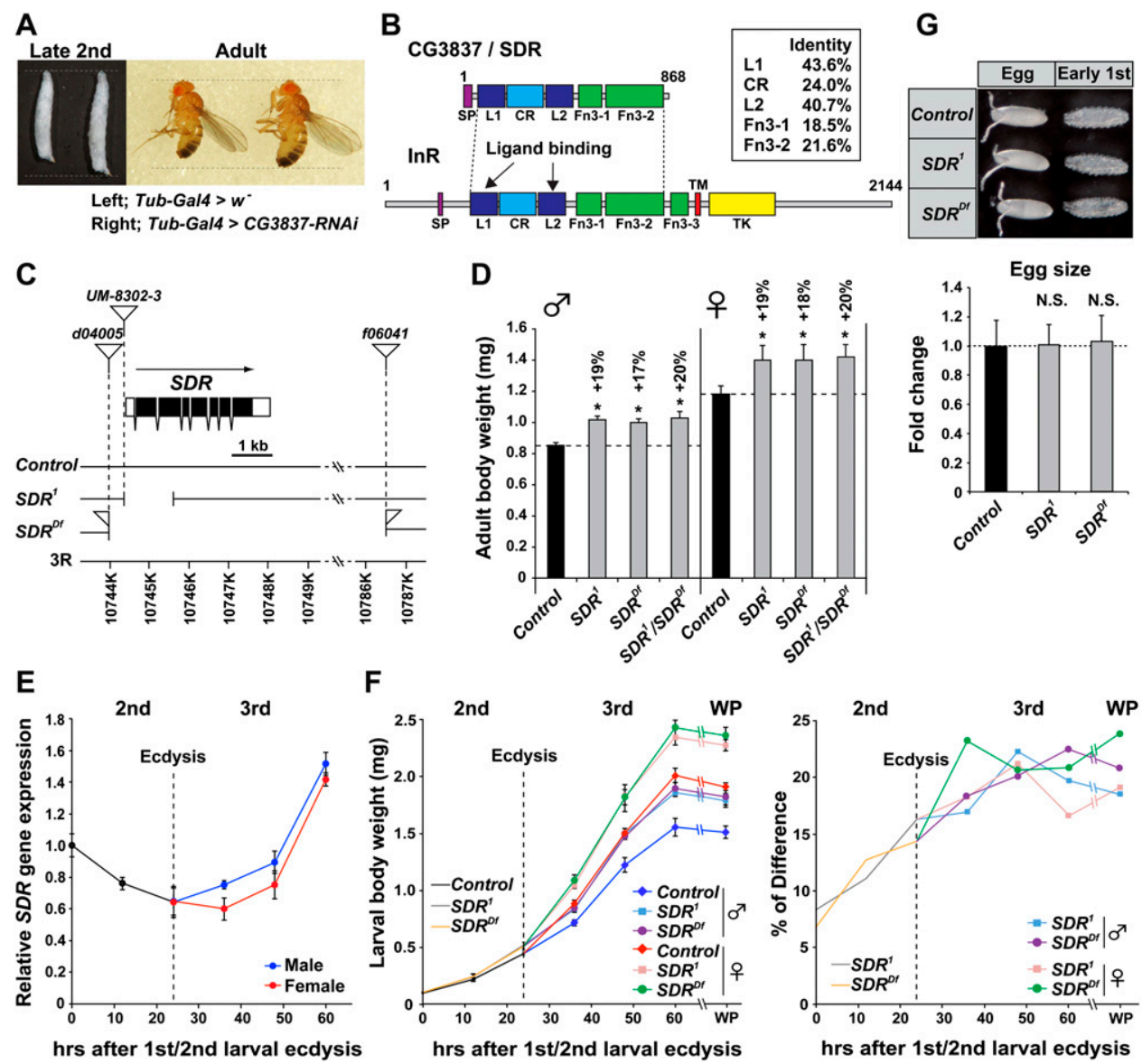

Figure 1. SDR negatively regulates body growth during the larval period. (A) Ubiquitous knockdown of CG3837 by Tub-Gal4 increases the size of larvae and adult flies. (B) Domain structure of CG3837/SDR and InR. Numbers refer to amino acids. (SP) Signal peptide; (L) L domain; (CR) furin-like cysteine-rich region; (Fn3) fibronectin type 3 domain; (TM) transmembrane domain; (TK) tyrosine kinase catalytic domain. $(C)$ Schematic representation of the $S D R$ locus and molecular nature of the mutants. The protein-coding regions and untranslated regions are represented by filled boxes and open boxes, respectively. The $P$-element insertion sites are marked with an inverted triangle. (D) $S D R$ mutant adult flies are heavier than control in both males and females. (E) $S D R$ is expressed throughout the larval period. $(F)$ Developmental changes in the wet weight of control and $S D R$ mutants. The percent difference in body weight between the control and $S D R$ mutants is shown on the right. (WP) White pupae. (G) The size of $S D R$ mutant eggs and newly hatched larvae is identical to that of control. All of the values are the mean and standard deviation (SD). $(n>4$ batches $[D, F], n>3$ batches $[E]$, or $n>15$ eggs $[G])$. (N.S.) Not significant; $\left(^{\star}\right) P<0.01$ (Student's $t$-test). 
difference in body weight reached $\sim 20 \%$ at the late third instar, which was comparable with the difference in adult body weight. These results indicate that SDR negatively regulates systemic growth during larval development. Notably, the expression levels of the major dilps during the larval period (dilp2, dilp3, and dilp5) were unchanged in the $S D R$ mutants (Supplemental Fig. S3B). Moreover, the neuronal morphology and the fluorescent signal of Dilp2 in brain insulin-producing cells (IPCs) were indistinguishable between control and SDR mutants (Supplemental Fig. S3C), indicating that SDR functions downstream from Dilp secretion by IPCs.

\section{Glia-derived SDR is critical to limit systemic growth}

SDR is a putative secreted protein. Indeed, SDR protein was detected in both cell lysates and culture medium when expressed in S2 cells (Fig. 2A). Consistent with this result, SDR was localized in vesicular structures in cells (Fig. 2B). To analyze endogenous SDR, we raised antibodies against SDR. These antibodies detected a protein of the expected molecular weight in cell-free circulating larval hemolymph (Fig. 2C). The corresponding band was abolished by RNAi-mediated knockdown of SDR, demonstrating the specificity of our antibodies and indicating that SDR is indeed secreted into the hemolymph.

We next analyzed the expression pattern of SDR and determined which tissue is the source of SDR. Quantitative RT-PCR (qRT-PCR) analyses revealed that SDR was highly expressed in the CNS in mid-third instar larvae (Fig. 2D). SDR transcripts were also detected in the midgut and in imaginal discs but not in the fat body, malpighian tubules, trachea, or salivary glands (data not shown). To corroborate the expression pattern of $S D R$, we constructed a Gal4 line under the control of a $3.5-\mathrm{kb}$ putative promoter region immediately upstream of the

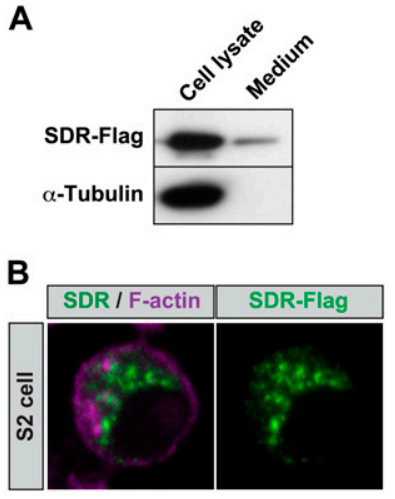

C
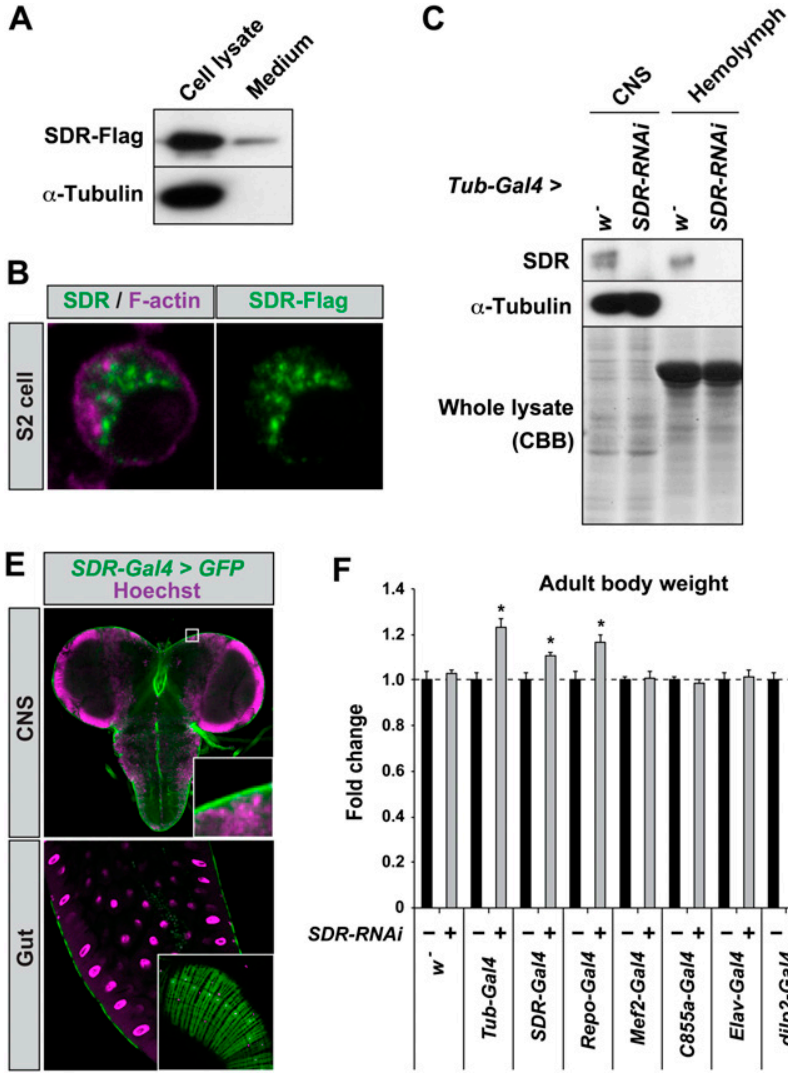

$\mathbf{F}$
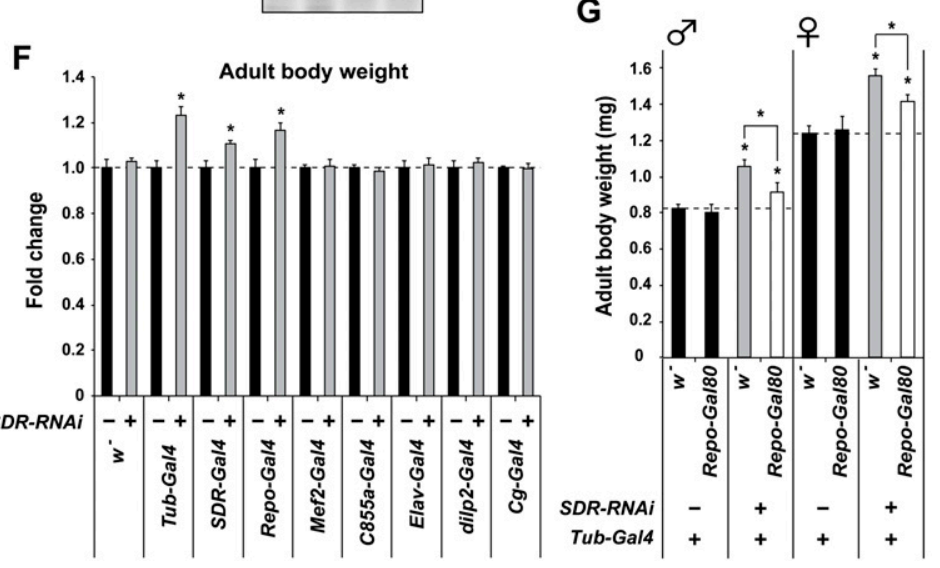

Figure 2. Glia-derived SDR regulates body growth non-cell-autonomously. (A) Flag-tagged SDR is secreted into cultured medium in S2 cells. The amount of SDR-Flag and $\alpha$-Tubulin was analyzed in S2 cell lysate and cell-free cultured medium. (B) SDR-Flag localizes in vesicular structures in S2 cells. $(C)$ SDR is a protein secreted into the hemolymph. The amount of SDR and $\alpha$-Tubulin was analyzed in a lysate of the CNS (brain-ventral ganglia complex) and cell-free hemolymph at the wandering stage. (Bottom) The amount of total proteins in the lysate was analyzed by CBB staining. $(D)$ Tissue distribution of $S D R$ transcripts was analyzed by qRT-PCR in mid-third instar larvae (24 h after second/third ecdysis). (FB) Fat body; (ID) imaginal discs; (MT) malpighian tubule. (E) SDR-Gal4 was expressed in surface glia and midgut muscles. The $S D R$-Gal4>UAS-CD8-GFP flies were immunostained for GFP (green) and nuclei (purple). Single confocal sections of the CNS and gut are shown. The inset in the CNS image shows the enlarged image of the boxed area. The inset in the Gut image shows the surface view of the midgut. $(F)$ Adult body weight was analyzed in tissue-specific knockdown of $S D R$. The Gal4 lines used were Tub (ubiquitous), Repo (glia), Mef2 (muscle), C855a (epithelia), Elav (neuron), dilp2 (IPC), and Cg (fat body). (G) The contribution of glia-derived SDR in the ubiquitous knockdown phenotype to body weight that is induced by Tub-Gal4>UAS-SDRRNAi. All of the values are the mean and SD $(n=3[D]$ or $n>4$ batches $[F, G]) .\left(^{\star}\right) P<0.01$ (Student's $t$-test). 
transcriptional start site (Supplemental Fig. S2). Specifically, CD8-GFP signal driven by SDR-Gal4 was detected in surface glia in the CNS and midgut muscles (Fig. 2E) but not in the imaginal discs (data not shown), suggesting that $S D R$-Gal4 partially mimics the endogenous expression pattern. Tissue-specific knockdown of $S D R$ confirmed that glia and the midgut musculature are the primary sources of $S D R$ transcript in the CNS and the gut, respectively (Supplemental Fig. S4).

To determine whether glia-derived SDR governs systemic growth defects, we silenced the expression of $S D R$ by using targeted RNAi. Knockdown of $S D R$ by $S D R$ Gal4 increased adult body weight (Fig. 2F). The knockdown of $S D R$ using a pan-glial Gal4 driver, Repo-Gal4, also increased body weight, while knockdown in muscles by Mef2-Gal4 or in epithelial cells, including imaginal discs by C855a-Gal4, did not (Fig. 2F). Other Gal4 lines that drive expression in neurons (Elav-Gal4), IPCs (dilp2Gal4), or the fat body (Cg-Gal4) did not affect body weight. However, the effects of Repo-Gal4 and SDRGal4 were less pronounced than those of Tub-Gal4, raising the possibility that other tissues contribute to the phenotype. To verify the critical role of glia-derived SDR, we suppressed $S D R$ in all tissues except glia by using Gal80, an inhibitor of Gal4. Repo-Gal80 partially suppressed the increase in adult body weight that was induced by Tub-Gal4>UAS-SDR-RNAi (Fig. 2G). These results suggest that surface glia are a major source of SDR in the regulation of systemic growth, although SDR is expressed in several other tissues, which may also contribute to growth regulation.

\section{SDR remotely controls the growth of peripheral tissues through IIS}

We next analyzed the proliferation of imaginal discs as a phenotypic readout of the IIS. Adult SDR mutant and knockdown flies exhibited larger wing size as a result of an increase in both cell size and cell number (Fig. 3A). The size difference of wing discs in the $S D R$ mutants was apparent during the late third instar larvae (Supplemental Fig. S5A,B). Although we detected $S D R$ expression in imaginal discs during the larval period, the phenotype of the adult wing was largely non-cell-autonomous because the targeted knockdown of $S D R$ in wing discs had no obvious effects on wing size (Supplemental Fig. S5C). Consistently, Repo-Gal80 suppressed the increase in the wing size that was induced by Tub-Gal4>UAS-SDR-RNAi (Supplemental Fig. S5D), indicating that glia secrete SDR to remotely control the growth of peripheral tissues.

In contrast, overexpression of SDR by Tub-Gal4 decreased adult body weight and led to decreased wing size (Fig. 3A,B; Supplemental Fig. S6A). Consistent with this result, we detected a difference in both cell size and cell number in the adult wing. Targeted expression of $S D R$ in glia, the fat body, and the muscles all resulted in increased SDR protein levels in the hemolymph (Fig. 3C) and was sufficient to reduce body weight and wing size (Fig. 3B). These findings support a non-cell-autonomous function of SDR in tissue growth.
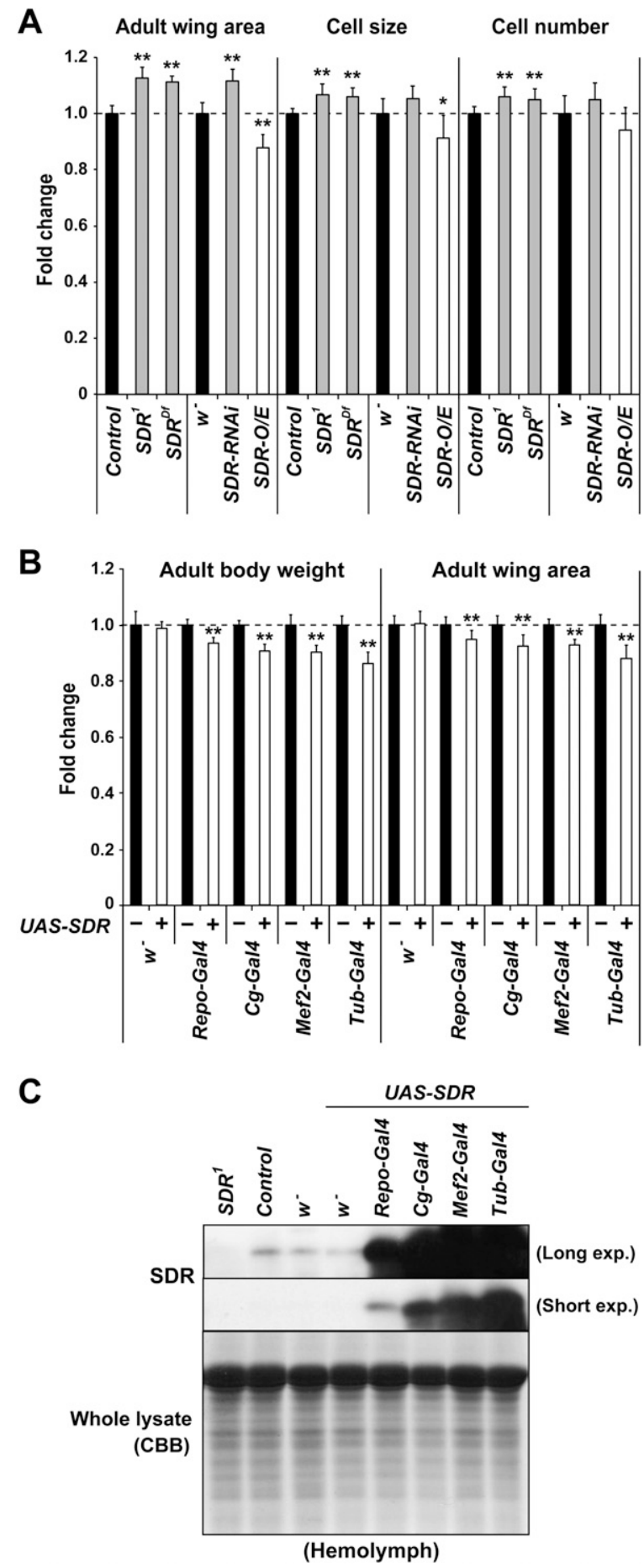

Figure 3. SDR remotely controls growth of peripheral tissue. (A) Wing area, cell size, and cell number were analyzed in SDR mutants and overexpressing flies. Tub-Gal4 was used for $S D R$ knockdown and overexpression. $(B)$ Adult body weight and wing area were analyzed in $S D R$ overexpression flies with the indicated driver. All of the values are the mean and SD $(n>10$ wings $[A], n>$ 4 batches, or $n>10$ wings $[B]) .\left(^{\star}\right) P<0.05$; $\left(^{\star \star}\right) P<0.01$. (C) SDR protein levels in the hemolymph were analyzed in $S D R$-overexpressing wandering larvae. The same membrane was exposed for a long time (long exp.) and a short time (short exp.) because of the highly enhanced expression of SDR by the Gal4/UAS system. 
One of the cytological readouts of IIS is the nuclear localization of dFoxo. dFoxo translocates from the cytoplasm into the nucleus when larvae experience nutrient deprivation or have decreased InR-dependent PI3K activity (Fig. 4A; Edgar 2006; Hietakangas and Cohen 2009). As expected, nuclear localization of dFoxo was indeed affected by the manipulation of $S D R$ levels in the fat body (Fig. 4A; Supplemental Fig. S7A,B). The effects of $S D R$ knockdown and overexpression on dFoxo localization were more apparent in larvae transiently grown on the restricted food that diminish IIS. To monitor PI3K activity, we also analyzed the membrane localization of $\mathrm{tGPH}$, a fusion protein of GFP to a pleckstrin homology (PH) domain of Grp1 (Britton et al. 2002). A slight but reproducible alteration in tGPH localization was observed following $S D R$ knockdown (Supplemental Fig. S7C).

When dFoxo is inactivated by an increase in IIS and excluded from the nucleus, dFoxo target genes such as Thor/4EBP, InR, and dilp6 are predicted to be downregulated (Puig et al. 2003; Slaidina et al. 2009). We therefore analyzed the expression of these genes as a molecular readout of IIS in the larval fat body and body wall, in which SDR expression level is low. Consistent with the changes that were observed in dFoxo localization, these genes were down-regulated in $S D R$ knockdown larvae (Fig. 4B; Supplemental Fig. S8). In contrast, $S D R$ overexpression resulted in an up-regulation of these genes, suggesting that IIS is affected in the peripheral tissues. The glia-specific manipulation of $S D R$ using Repo-Gal4 resulted in less pronounced but consistent changes in gene expression (Supplemental Fig. S8). Taken together, these results strongly suggest that $S D R$ controls IIS in peripheral tissues.

\section{SDR genetically interacts with IIS in vivo}

If SDR negatively regulates the activity of Dilps through InR, then a reduction in Dilp or InR levels should suppress the $S D R$ mutant phenotype. Indeed, the heterozygous InR mutant slightly suppressed the $S D R$ knockdown phenotype (Supplemental Fig. S6B). Furthermore, the expression of dominant-negative $\operatorname{In} R(\operatorname{InR}-D N)$ was largely epistatic to the $S D R$ knockdown phenotype and led to small body size (Fig. 4C).

PTEN negatively regulates intracellular InR signaling to limit cellular growth and should therefore exhibit positive genetic interactions with $S D R$. The ubiquitous knockdown of PTEN increased body size to an extent similar to the loss of SDR (Fig. 4C). The double knockdown of PTEN and SDR further increased body size. In contrast, the loss of PTEN rendered flies less sensitive to the effect of size decrease by SDR overexpression, which is consistent with the notion that SDR acts upstream of PTEN (Fig. 4C).

We also observed negative genetic interactions between $S D R$ and dilps. First, reduction in dilp levels partially suppressed the $S D R$ knockdown phenotype (Supplemental Fig. S6B). Second, the effects of SDR overexpression were completely suppressed by coexpression with dilp3 (Supplemental Fig. S6C). Overexpression of dilp2 caused embryonic lethality (Brogiolo et al. 2001), while overexpression of dilp5 was semilethal at the larval stage with only a few escapers. Although the coexpression of $S D R$ failed to rescue embryos overexpressing dilp2, larvae overexpressing dilp5 exhibited significant phenotypic rescue (Supplemental Fig. S6D). Consistent with this result, SDR knockdown led to complete lethality in larvae overexpressing dilp5. Altogether, these
A

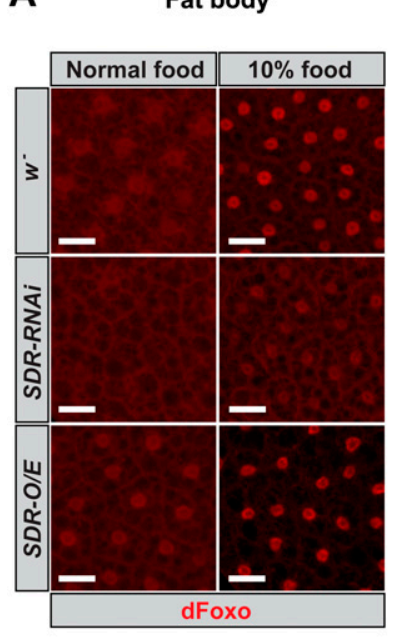

B

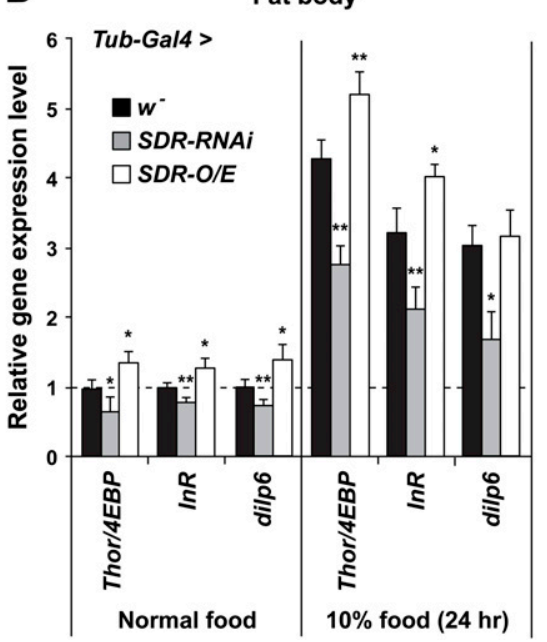

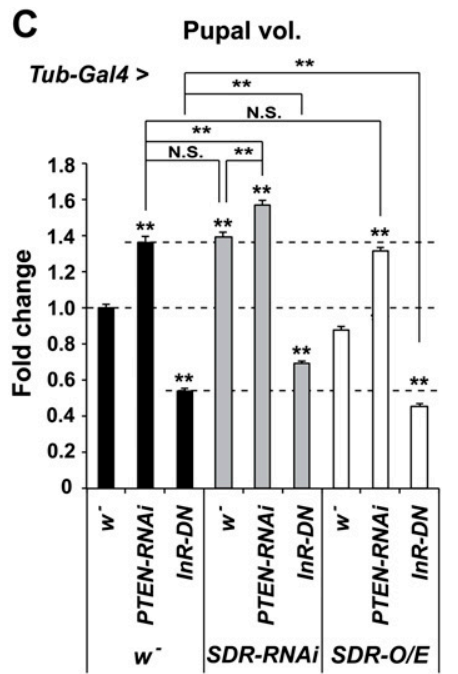

Figure 4. SDR controls body growth through InR signaling in vivo. $(A)$ dFoxo localization was analyzed in the fat body of mid-third instar larvae. Early third instar larvae just after molting were grown on normal food conditions (normal food) or adverse food conditions (10\% food contents) for $24 \mathrm{~h}$. Tub-Gal4 was used for the manipulation of SDR levels. Bars, $25 \mu \mathrm{m}$. (B) Thor/4EBP, InR, and dilp6 transcripts were analyzed by qRT-PCR in the sample of $A$. The values are the mean and SD $(n=3-4)$. (C) SDR genetically interacts with InR signaling in vivo. The volume of pupae was analyzed in flies of the indicated genotypes. The values are the mean and SEM $(n=40)$. $\left.\left(^{\star}\right) P<0.05 ;{ }^{\star \star}\right) P<0.01$ (Student's $t$-test). 
genetic data demonstrate that $S D R$ negatively interacts with dilps in vivo to inhibit IIS and systemic growth.

\section{SDR interacts with several Dilps independent of Imp-L2 in vitro}

The sequence similarity between SDR and InR raises the possibility that SDR physically interacts with Dilps. To test this possibility, we systematically analyzed the binding of SDR to the seven Dilps in vitro. Because ImpL2 has been shown to selectively bind to Dilp2 and Dilp5 but not to Dilp3 (Arquier et al. 2008; Honegger et al. 2008; Alic et al. 2011), we included Imp-L2 in our analysis as a control and to ascertain its interaction with the other Dilps. Flag-tagged SDR was cotransfected into HEK293T cells with each of the His-tagged Dilps, and the cell lysates were incubated with nickel agarose. As expected, SDR was coprecipitated with the Dilps (Fig. 5A). Specifically, Dilp3 showed the highest recovery of SDR, and Dilp1, Dilp2, Dilp5, Dilp6, and Dilp7 showed modest recovery of SDR. Under these conditions, Imp-L2 was strongly coprecipitated with Dilp1, Dilp2, Dilp4, Dilp5, and Dilp6, whereas Dilp3 and Dilp7 showed low recovery of Imp-L2. The interaction of SDR and Imp-L2 with the Dilps was found to be specific because a potent growth regulator, Idgf2, did not interact with any of the Dilps (Supplemental Fig. S9A). In addition, the binding of SDR to the Dilps was confirmed by reverse pull-down of Flag-tagged Dilps with His-tagged SDR (Supplemental Fig. S9C).

Drosophila ALS has been reported to form an evolutionarily conserved ternary complex with Dilp2 and ImpL2 (Arquier et al. 2008). However, we were unable to detect an interaction between SDR and either Imp-L2 or ALS (Fig. 5B; Supplemental Fig. S9D,E). Furthermore, Imp-L2 was able to compete out the interaction between
SDR and Dilp2 (Fig. 5C), indicating that Imp-L2 and SDR bind to Dilp2 in a mutually exclusive manner. This result, together with our finding that SDR and Imp-L2 display differential affinities for the different Dilps (Fig. $5 \mathrm{~A})$, suggests that SDR forms a protein complex with the Dilps independent of the Imp-L2-ALS complex.

To further confirm the physical interaction between SDR and Dilps in solution, we performed pull-down experiments using secreted proteins in culture medium from transfected HEK293T cells. Although the secretion efficiency and/or stability of SDR in medium were very low, the interaction of SDR and Dilp3 was detected (Supplemental Fig. S9B). Imp-L2 was readily secreted into the medium and consistently interacted with Dilp1, Dilp2, Dilp4, Dilp5, and Dilp6. These results indicate that the interaction occurs not only inside intracellular vesicles, such as the ER-Golgi network, but also outside of cells. Nevertheless, our in vitro analysis is quite distinct from the natural context; these interactions possibly take place in the hemolymph. Therefore, we cannot exclude the possibility that post-translational modification and/or processing affect the binding affinity between SDR/Imp-L2 and Dilps in vivo.

\section{Secretion of SDR into the hemolymph is independent of nutritional status}

The secretion of each Dilp into the hemolymph is regulated in response to nutrient levels. Specifically, the secretion of Dilp2, Dilp3, and Dilp5 from brain IPCs and the expression of $A L S$ in the fat body are suppressed by starvation (Colombani et al. 2003; Géminard et al. 2009). In contrast, starvation up-regulates the expression of dilp6 and Imp-L2 in the fat body under adverse nutritional conditions (Honegger et al. 2008; Slaidina et al. 2009). To address how SDR cooperates with Imp-L2 and ALS to
A
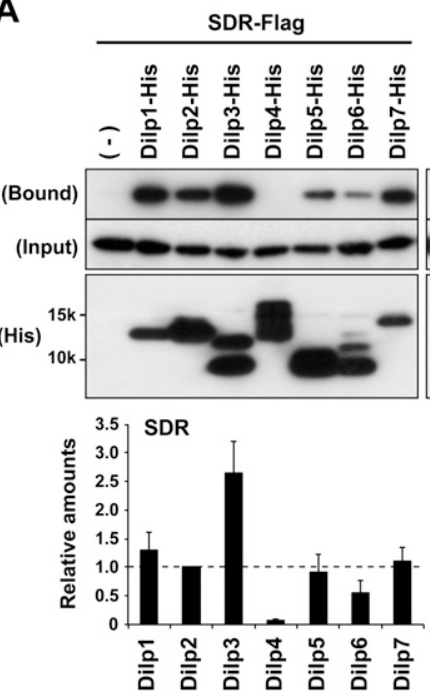

Imp-L2-Flag
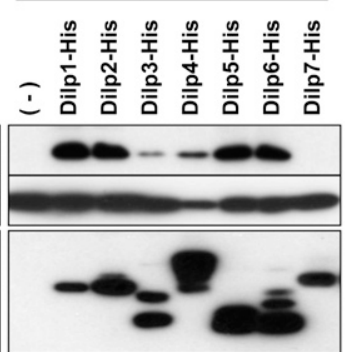

1.6 Imp-L2

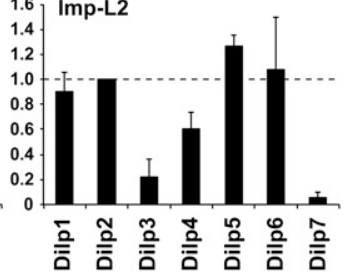

B

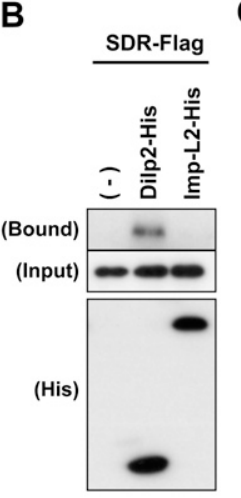

C

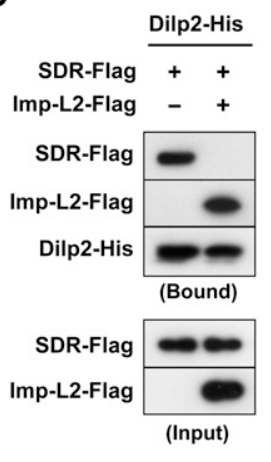

Figure 5. SDR interacts with Dilps independent of Imp-L2. (A) SDR and Imp-L2 bind to Dilps with different binding affinities in HEK293T cells. Flag-tagged proteins were analyzed in the precipitates of His-tagged Dilps in transfected cell lysate. The relative amounts of the precipitated SDR and Imp-L2 were normalized to input levels. The signal was further normalized to the value of Dilp2His (set to 1). (B) SDR binds to Dilp2 but not to Imp-L2. (C) Imp-L2 competes for binding between SDR and Dilp2. 
regulate the activity of circulating Dilps in response to nutritional status, we analyzed the expression and secretion levels of SDR under different nutrient conditions. As expected, $\operatorname{In} R$ was up-regulated, while dilp5 was downregulated, by starvation (Fig. 6A; Ikeya et al. 2002; Puig et al. 2003). These changes were completely reversed by refeeding. However, under identical conditions, SDR expression was relatively constant regardless of feeding state (Fig. 6A). These results were further confirmed by analyzing the $S D R$ expression in individual tissues (Supplemental Fig. S10). In addition, SDR protein levels in the hemolymph were relatively invariant (Fig. 6B), indicating that the secretion of SDR is constitutive.

\section{SDR is critical for survival under adverse food conditions}

If SDR limits circulating Dilp activity independent of nutrient levels, the loss of $S D R$ should have growthpromoting effects even under limiting nutrient conditions.
To test this possibility, $S D R$ mutants and control first instar larvae were grown at a defined density in vials that contained various concentrations of yeast (the major source of protein in fly food). SDR mutants increased in body size regardless of the nutrient conditions (Fig. 6C). Considering that $S D R$ knockdown inhibits dFoxo activity in transient adverse food conditions (Fig. 4A,B), SDR constitutively regulates Dilp activity irrespective of nutrient status.

Because IIS coordinates nutritional status with growth and metabolism during development (Edgar 2006; Hietakangas and Cohen 2009), SDR mutants may suffer from a balance of growth and metabolism by impaired IIS. When control larvae were grown on a diet with even lower nutrient levels, such that the flies received only $10 \%$ of the normal amount of food, they were significantly delayed in pupation and exhibited a higher mortality rate during the larval feeding period (Fig. 6D,E). SDR mutants underwent puparium formation to a degree similar to the control lines under these conditions. However, more than half of the
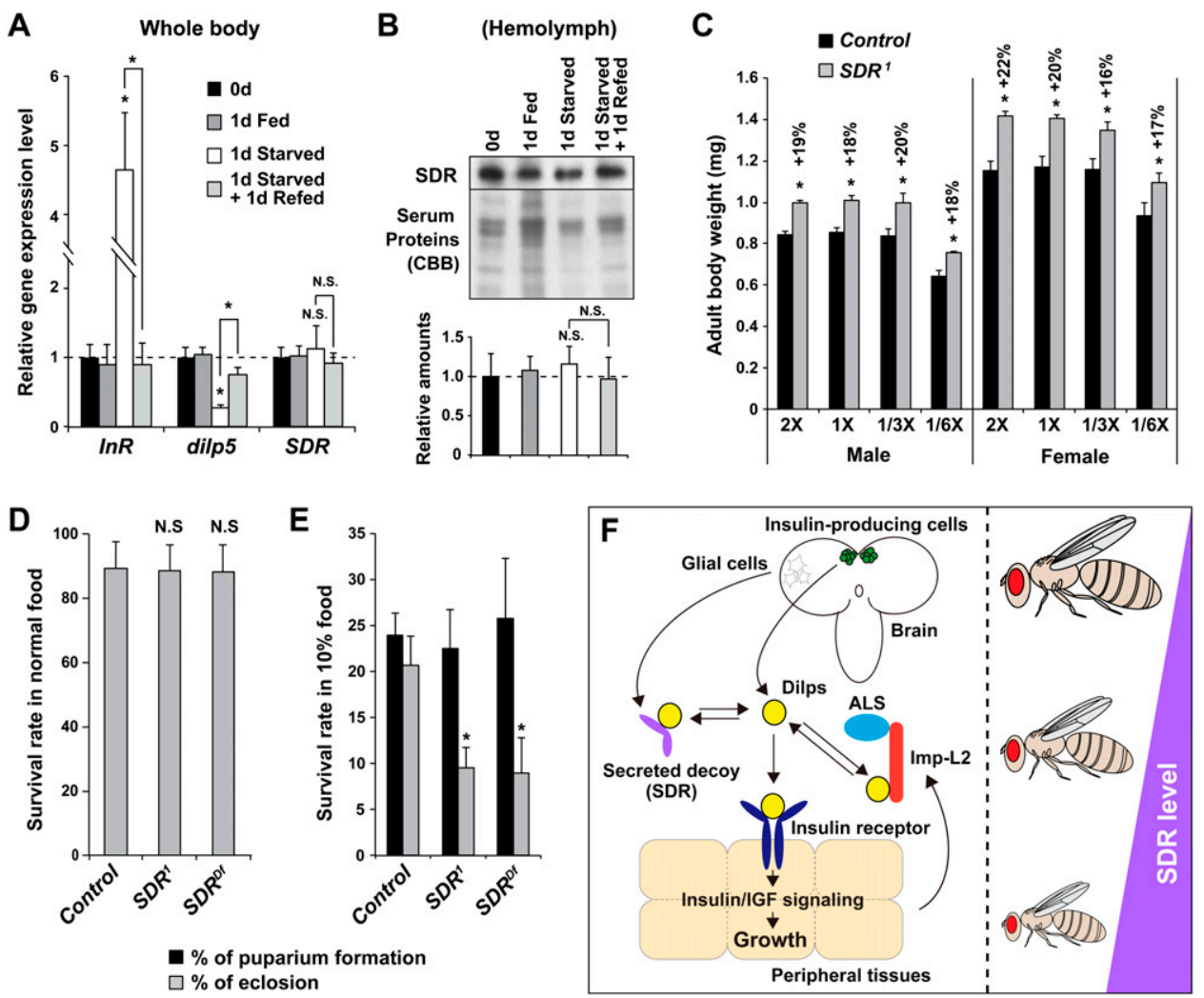

Figure 6. SDR is constitutively secreted in the hemolymph and is critical under adverse food conditions. (A) InR, dilp5, and $S D R$ expression levels were analyzed by qRT-PCR in different food conditions. Early third instar larvae just after molting were used (set to $0 \mathrm{~d})$. (B) Constitutive production of SDR into the hemolymph. SDR protein levels in the hemolymph were analyzed in the sample shown in $A$. (C) $S D R$ mutants promote body growth regardless of the nutrient conditions. The relative concentrations of yeast contents in food are indicated $(2 \times, 1 \times, 1 / 3 \times$, and $1 / 6 \times) .(D, E) S D R$ mutants grown on the restricted food conditions exhibit an elevated mortality rate during metamorphosis. A defined number of newly hatched first instar larvae was seeded on normal food $(D)$ or lownutrient food ( $10 \%$ food) $(E)$, and the number of pupae (puparium formation) and eclosed flies (eclosion) was counted. All of the values are the mean and $\mathrm{SD}(n=3[A, B], n>4$ batches $[C]$, or $n=7-10$ vials $[D, E]) .\left(^{\star}\right) P<0.01$ (Student's $t$-test). (F) A proposed model showing the role of SDR in growth control. Glia-derived SDR binds to circulating Dilps in the hemolymph and negatively regulates systemic growth by antagonizing insulin/IGF signaling in peripheral tissues. The function of SDR is likely complementary to and redundant with that of Imp-L2. 
SDR mutant pupae eventually died during metamorphosis. The survival of Imp-L2 mutant larvae is consistently compromised under starvation conditions but not under normal food conditions (Honegger et al. 2008). Taken together, these results suggest that $S D R$ is essential for limiting systemic growth by adjusting IIS under adverse food conditions.

\section{Discussion}

\section{The importance of SDR in growth regulation}

Drosophila has seven Dilps and one IGFBP-type protein (Imp-L2), whereas mammals have seven IGFBPs. An ongoing challenge has been to resolve how these proteins cooperate in the control of systemic growth via IIS. Our biochemical experiments revealed that Imp-L2 binds to several Dilps, including Dilp1, Dilp2, Dilp4, Dilp5, and Dilp6. In contrast, SDR binds most strongly to Dilp3, indicating that each Dilp has distinct binding preferences for either Imp-L2 or SDR. Interestingly, in addition to expression in IPCs, dilp3 is expressed in a subset of glia and midgut muscles where $S D R$ is highly expressed (Veenstra et al. 2008; Sousa-Nunes et al. 2011). Although SDR can be detected in the hemolymph and regulates systemic growth, SDR may also act locally in the tissues where it is expressed. The slight up-regulation of the $S D R$ transcripts during the third instar likely reflects $S D R$ expression in imaginal discs (data not shown). It is possible that $S D R$ expression is actively regulated in a stage- and tissue-specific manner to fulfill such a local function.

Recent reports have revealed that fluctuations in ligand levels have more significant biological impacts on downstream signaling events than was previously appreciated (Kubota et al. 2012). The dynamics of insulin levels and their specific temporal pattern can elicit a unique physiological response through different kinetic behavior and network connectivity of Akt. Therefore, the function of SDR and Imp-L2 in the regulation of Dilps may be more complex than the interference of a ligand-receptor interaction. The ability to maintain constant Dilp-binding protein levels in the hemolymph would provide a robust system for growth regulation in combination with dynamic Imp-L2 levels.

\section{Similarities and differences between SDR and the Imp-L2-ALS complex}

There are functional similarities between SDR and ImpL2 in the sense that both act as negative regulators of IIS (Fig. 6F). However, our phenotypic and biochemical analyses revealed important differences between SDR and the Imp-L2-ALS complex (Arquier et al. 2008; Honegger et al. 2008). First, heterozygous $S D R$ mutants exhibited approximately normal body size (data not shown), whereas loss of one copy of Imp-L2 leads to a moderate increase in body size. The partial knockdown of $S D R$ by a weak ubiquitous Gal4 driver, arm-Gal4, consistently showed no phenotype (data not shown). Second, overexpression of $I m p-L 2$ causes lethality, whereas moderate overexpression of $I m p-L 2$ significantly impairs body growth, resulting in a delay of larval development. In contrast, $S D R$ overexpression led to moderate reduction in body weight with no apparent developmental delay or lethality, even though the vast excess of SDR proteins was achieved compared with endogenous levels. Consistently, the lethality induced by ectopic Dilp2 expression can be rescued by overexpression of Imp-L2 but not by overexpression of $S D R$. Third, both Imp-L2 and ALS are widely expressed in a number of different tissues. Fat body-derived Imp-L2-ALS, however, seems to be critical for the systemic regulation of IIS, whereas glia-derived SDR is important in this respect. Last, Imp-L2 and $A L S$ expression responds to nutritional status, whereas the production of SDR is constant.

It is interesting to consider the analogous case in mammals, which exhibit distinct alterations in IGFBP protein levels after fasting; IGFBP1 is up-regulated by fasting, whereas IGFBP2 and IGFBP3 remain constant in circulating blood (Thissen et al. 1994). SDR seems to act as a constitutive regulator of Dilps in the hemolymph, whereas Imp-L2 is a dynamic regulator that inhibits IIS in response to nutrient levels. It is equally possible, however, that the function of SDR is regulated post-translationally in the hemolymph; secreted SDR is inactive, and modifications and/or binding partners allow SDR to bind to Dilps. In contrast, Imp-L2 is likely active once secreted into the hemolymph. Further analysis will be required to understand the regulatory mechanism of SDR in the hemolymph and the functional relationship between SDR and the Imp-L2-ALS complex. Together, these observations suggest that Drosophila uses two different regulators that have distinct molecular activities to fine-tune the activity of circulating Dilps.

\section{The conservation of a soluble decoy system for regulating IIS}

In mammals, antagonistic soluble decoys have been described for many receptors, including receptor tyrosine kinases, immune receptors, and seven-pass transmembrane receptors (Mantovani et al. 2001; Jones and Jomary 2002; Vorlová et al. 2011). Although the SDR-like gene is found only in dipterous insects, including flies and mosquitoes ( $\mathrm{N}$ Okomoto and $\mathrm{T}$ Nishimura, unpubl.), similar decoy systems for IIS are likely present in other species. The Caenorhabditis elegans insulin receptor Daf-2 contains an alternative splice variant that encodes a putative secreted protein (WormBase, http://www. wormbase.org). Similarly, the mammalian insulin receptor can potentially produce a soluble decoy by alternative intronic polyadenylation (Vorlová et al. 2011). In both cases, the physiological function of the putative secreted protein has not been addressed. The type II IGF receptor, also known as mannose-6-phosphate receptor, is thought to be cleaved to produce a soluble form (sIGF2R) that binds to IGF-II with high affinity in vivo (Valenzano et al. 1995). Indeed, ectopic expression of sIGF2R inhibits cellular growth and reduces organ size (Scott et al. 1996; Zaina and Squire 1998). In Drosophila, however, a soluble 
form of InR that is produced by alternative splicing or ectodomain shedding has not been described. Instead, SDR may have arisen by a gene duplication event in Drosophila.

It remains unknown whether SDR can form a nonfunctional heterodimer with InR on the plasma membrane and thereby directly antagonize signaling through InR. Based on sequence similarity, SDR and InR are expected to show similar binding affinities for each Dilp. We therefore hypothesize that receptor-like decoy molecules function to fine-tune receptor signaling by sequestering multiple ligands. The constitutive production of such decoys may be beneficial to adapt endocrine signals in response to environmental changes, including the availability of food.

\section{Materials and methods}

\section{Fly strains}

The following stocks were used: UAS-Dilp2, UAS-Dilp3, UAS-Dilp5 (from E. Hafen), dilp2-Gal4 (from E. Rulifson), repo-Gal80 (from T. Awasaki), UAS-CG3837-RNAi (from the Bloomington Drosophila Stock Center, no. 35277), UAS-CG3837 (P\{GSV6\}GS15193; the Kyoto Drosophila Stock Center, no. 206122), and UAS-PTENRNAi (the Vienna Drosophila RNAi Center, no. 35731).

The following stocks were obtained from the Bloomington Drosophila Stock Center: Df(3L)Ilp2-3, Df(3L)Ilp1-4, Ilp5 ${ }^{4}$, $I l p 6^{68}, \ln { }^{05545}$, tub-Gal4, repo-Gal4, Mef2-Gal4, Cg-Gal4, elav-Gal4, C855a-Gal4 (used as a imaginal disc driver), en-Gal4, UAS-dInR-K1409A, tGPH, and UAS-mCD8-GFP.

\section{Generation of SDR mutants and antibodies}

A transposable $P$-element insertion, $P\{R S 3\} U M-8302-3$, was used to generate $C G 3837 / S D R$ mutants by imprecise excision. The progeny were first screened for the loss of an eye color marker $\left(w^{+}\right)$, and the extent of the deletion in each mutant was determined by PCR and subsequent DNA sequencing. $S D R^{1}$ is a deletion of 1191 base pairs (bp) spanning the region from $-65 \mathrm{bp}$ to $+1126 \mathrm{bp}$ relative to the predicted transcriptional start site. The $S D R^{D f}$ mutant was derived from $P\{X P\} d 04005$ and $p B a c\{W H\} f 06041$ using the strategy described in a previous study (Parks et al. 2004). The intrachromosomal deletion was confirmed by PCR, and the line was backcrossed to the $w^{-}$control strain four times. Both mutants were maintained as homozygous stocks.

SDR antibodies were generated in rabbits against the peptide SGPQPDDEDYYNKEQITC and affinity-purified.

\section{Starvation experiments}

The animals were reared on fly food that contained, per liter $(1 \times$ recipe), $8 \mathrm{~g}$ of agar, $100 \mathrm{~g}$ of glucose, $45 \mathrm{~g}$ of dry yeast, $40 \mathrm{~g}$ of corn flour, $4 \mathrm{~mL}$ of propionic acid, and $0.45 \mathrm{~g}$ of butylparaben (in ethanol). For the transient starvation experiments, early third instar larvae (0-6 h after second/third larval ecdysis) were washed in PBS and transferred to either a new fly food (fed sample) or a vial that contained $0.8 \%$ agar in PBS (fasted sample). For the low-nutrient challenge, the results of which are presented in Figures 4, A and B, and 6E, 10\% food was prepared by diluting the $1 \times$ recipe food with $0.8 \%$ agar in PBS. For the experiments that are shown in Figure 6C, fly food that contained either an increased or reduced amount of dry yeast was used $(2 \times$, $1 / 3 \times$, or $1 / 6 \times$ the amount used for the $1 \times$ recipe). No yeast paste was added to the fly tubes for any of the experiments.

\section{Hemolymph sample preparation}

Larvae were collected, rinsed in PBS, and dried on tissue paper. The cuticle was carefully torn to release the hemolymph on a parafilm membrane. The total hemolymph of 10 larvae was collected by micropipette, diluted four times with a buffer $(5 \mathrm{mM}$ Tris- $\mathrm{HCl}$ at $\mathrm{pH} 6.6,137 \mathrm{mM} \mathrm{NaCl}, 2.7 \mathrm{mM} \mathrm{KCl}$ ), and then cleared by centrifugation. The supernatant was boiled in SDS sample buffer and subjected to SDS-PAGE analysis.

\section{Quantification of weight and volume}

Developmental staging and quantification of weight were performed as previously described (Okamoto et al. 2009). The volumes of pupae and eggs were measured using the formula $4 / 3 \pi(L / 2)(1 / 2)^{2}$ (where $L$ is length and 1 is diameter).

\section{Plasmid construction}

The cDNAs encoding dilp1-7, Idgf2, Imp-L2, ALS, and CG3837/ $S D R$ were cloned by RT-PCR using sequenced strains obtained from the Bloomington Stock Center. All PCR fragments were validated by DNA sequencing. Full-length ORFs without stop codons were subcloned into modified pUAST vectors containing a C-terminal Flag tag. For mammalian expression, modified pCAGGS vectors containing a C-terminal Flag or His tag were used.

The SDR-promoter-Gal4 (SDR-Gal4) line was generated by cloning a $3.5-\mathrm{kb}$ PCR fragment containing an upstream sequence from the transcriptional start site into pPT-Gal4 (Sharma et al. 2002). Transgenic flies were generated by a standard injection procedure (BestGene, Inc.).

\section{Immunocytochemistry}

Larval tissues were dissected in PBS and fixed for $10 \mathrm{~min}$ in $3.7 \%$ formaldehyde in PBS containing $0.2 \%$ Triton X-100 (PBST). After several washes in PBST, the tissues were blocked in PBST containing $2 \% \mathrm{BSA}$ for at least $30 \mathrm{~min}$ at room temperature. The tissues were then incubated with primary antibodies overnight at $4^{\circ} \mathrm{C}$. After washing multiple times in PBST, the tissues were incubated with secondary antibodies for $1-2 \mathrm{~h}$ at room temperature. After several washes, the tissues were mounted in $50 \%$ glycerol. The primary antibodies used were mouse anti-Flag (Sigma), chick anti-GFP (Abcam), rabbit anti-Dilp2 (Okamoto et al. 2012), and rabbit anti-dFoxo antibodies (from P. Léopold). Alexa-conjugated (Alexa488 or Alexa555) secondary antibodies and phalloidin (Invitrogen) were used. The nuclei were stained with Hoechst 33342.

\section{Image analysis}

Images were acquired on a Zeiss LSM700 confocal microscope and were processed in Photoshop (Adobe Systems). The sizes of adult wings and larval imaginal discs were determined using LSM Image Browser software (Zeiss). Cell density was analyzed by counting the number of wing hairs in a $0.01-\mathrm{mm}^{2}$ area of the wing (Brogiolo et al. 2001). The anterior region (the area between the wing margin and L2) and the posterior region (the area between L3 and the wing margin) were measured to compare the size of the different wing compartments.

For the quantification of dFoxo localization, the average fluorescence intensity per area was determined in the region of the nucleus and the cytoplasm in the same cells using LSM Image Browser software. All of the compared images were acquired under identical parameters on the same day. The data 
represent the mean and standard deviation of 20-25 cells from three to four samples in each condition. Statistical significance was determined using Student's two-tailed $t$-test.

\section{RT-qPCR analysis}

Total RNA was prepared by using an RNeasy minikit (Qiagen) and RNase-Free DNase set (Qiagen), and reverse transcription was performed using PrimeScript RT reagent kit (Takara Bio). qRT-PCR was performed on an ABI PRISM 7500 Real-Time PCR system (Applied Biosystems) using SYBR Premix Ex TaqII (Takara Bio). The transcript levels of target mRNA sequences were normalized with rp49 levels in the same samples. The primers used were as follows: $S D R$ sense primerl (N terminus), 5'-TATCTAATGGGCAATGCCACTCGGC-3'; SDR antisense primer1 (N terminus), 5'-AGGAGTTCCGGATGAACTCGAA GTC-3'; SDR sense primer2 (C terminus), 5'-CACGACTATCG CAGAAAGGAGTTCG-3'; SDR antisense primer2 (C terminus), 5'-AATTGCGGCCAATCTCTCCTTAGCC-3'; Thor/4EBP sense primer, 5'-TGATCACCAGGAAGGTTGTCATCTC-3'; Thor/ $4 E B P$ antisense primer, 5'-GAGCCACGGAGATTCTTCATGA AAG-3'; InR sense primer, 5' -TGAGCATGTGGAGCACATCAA GATG-3'; and InR antisense primer, 5'-CGTAGGAGATTTTC TCGTTTGGCTG-3'. The primers used to detect dilp levels have been reported previously (Okamoto et al. 2009).

\section{Cell culture and transfection}

S2 cells were cultured with Schneider's medium supplemented with $10 \%$ fetal bovine serum (FBS), penicillin, and streptomycin. Transfection was carried out using Effectene (Qiagen), and cultured medium and cells were collected $2 \mathrm{~d}$ after transfection. Cell-free cultured medium was obtained by taking supernatant following centrifugation at $3000 \mathrm{rpm}$ for $10 \mathrm{~min}$. HEK293T cells were cultured in DMEM with $10 \%$ FBS, and transfection was performed with polyethyleneimine (Polysciences).

\section{Binding assays}

For the pull-down assays, HEK293T cells transfected with the intended plasmids in six-well plates were extracted with lysis buffer $(20 \mathrm{mM}$ Tris- $\mathrm{HCl}$ at $\mathrm{pH} 7.5,150 \mathrm{mM} \mathrm{NaCl}, 2 \%$ glycerol, $20 \mathrm{mM}$ imidazole, $1 \%$ Triton X-100, $50 \mu \mathrm{g} / \mathrm{mL}$ PMSF, Complete Protease Inhibitor Cocktail [Roche]) and cleared by centrifugation at $15,000 \mathrm{rpm}$ for $10 \mathrm{~min}$. For the pull-down assays in solution, conditioned medium from transfected HEK293T cultures was collected, cleared by centrifugation at $3000 \mathrm{rpm}$ for 5 min, and then mixed with $1 / 5 \mathrm{vol}$ of lysis buffer. The cell lysates or cultured medium were incubated with Ni-NTA agarose (Qiagen) for $2 \mathrm{~h}$ at $4^{\circ} \mathrm{C}$. The affinity-purified protein complex was washed three times with lysis buffer and eluted by boiling in SDS sample buffer. We used mammalian HEK293T cells, given that dilp6 is expressed endogenously in Drosophila S2 cells at high levels (FlyAtlas, http://www.flyatlas.org; data not shown), which may have affected the results of the binding assay and may make interpretation difficult.

Antibodies used in Western blotting were mouse anti- $\alpha$-Tubulin, mouse anti-Flag (Sigma), mouse anti-RGS-6xHis (Qiagen), and mouse anti-HA (MBL).

\section{Acknowledgments}

We thank T. Awasaki, E. Hafen, P. Léopold, E. Rulifson, Y. Sharma, the Bloomington Stock Center, the Kyoto Stock Center, and the Vienna Drosophila RNAi Center for fly stocks; members of the Hayashi, Kuranaga, and Matsuzaki laboratories for their valuable discussion; members of the Nishimura laboratory for their technical help; and R. Niwa, F. Wirtz-Peitz, and N. Yamanaka for critical reading of the manuscript. This work was supported in part by a Grant-in-Aid for Scientific Research from the Ministry of Education, Culture, Sports, Science, and Technology of Japan (to N.O. and T.N.). N.O is supported by a RIKEN Special Post-Doctoral Fellowship.

\section{References}

Alic N, Hoddinott MP, Vinti G, Partridge L. 2011. Lifespan extension by increased expression of the Drosophila homologue of the IGFBP7 tumour suppressor. Aging Cell 10: 137147.

Arquier N, Géminard C, Bourouis M, Jarretou G, Honegger B, Paix A, Léopold P. 2008. Drosophila ALS regulates growth and metabolism through functional interaction with insulinlike peptides. Cell Metab 7: 333-338.

Britton JS, Lockwood WK, Li L, Cohen SM, Edgar BA. 2002. Drosophila's insulin/PI3-kinase pathway coordinates cellular metabolism with nutritional conditions. Dev Cell 2: 239249.

Brogiolo W, Stocker H, Ikeya T, Rintelen F, Fernandez R, Hafen E. 2001. An evolutionarily conserved function of the Drosophila insulin receptor and insulin-like peptides in growth control. Curr Biol 11: 213-221.

Colombani J, Raisin S, Pantalacci S, Radimerski T, Montagne J, Léopold P. 2003. A nutrient sensor mechanism controls Drosophila growth. Cell 114: 739-749.

Edgar BA. 2006. How flies get their size: Genetics meets physiology. Nat Rev Genet 7: 907-916.

Géminard C, Rulifson EJ, Léopold P. 2009. Remote control of insulin secretion by fat cells in Drosophila. Cell Metab 10: 199-207.

Hietakangas V, Cohen SM. 2009. Regulation of tissue growth through nutrient sensing. Annu Rev Genet 43: 389-410.

Honegger B, Galic M, Köhler K, Wittwer F, Brogiolo W, Hafen E, Stocker H. 2008. Imp-L2, a putative homolog of vertebrate IGF-binding protein 7, counteracts insulin signaling in Drosophila and is essential for starvation resistance. I Biol 7: 10.

Hwa V, Oh Y, Rosenfeld RG. 1999. The insulin-like growth factor-binding protein (IGFBP) superfamily. Endocr Rev 20: 761-787.

Ikeya T, Galic M, Belawat P, Nairz K, Hafen E. 2002. Nutrientdependent expression of insulin-like peptides from neuroendocrine cells in the CNS contributes to growth regulation in Drosophila. Curr Biol 12: 1293-1300.

Jones SE, Jomary C. 2002. Secreted Frizzled-related proteins: Searching for relationships and patterns. Bioessays 24: 811820.

Kubota H, Noguchi R, Toyoshima Y, Ozaki Y, Uda S, Watanabe K, Ogawa W, Kuroda S. 2012. Temporal coding of insulin action through multiplexing of the AKT pathway. Mol Cell 46: $820-832$.

Mantovani A, Locati M, Vecchi A, Sozzani S, Allavena P. 2001. Decoy receptors: A strategy to regulate inflammatory cytokines and chemokines. Trends Immunol 22: 328-336.

Nakae J, Kido Y, Accili D. 2001. Distinct and overlapping functions of insulin and IGF-I receptors. Endocr Rev 22: 818-835.

Okamoto N, Yamanaka N, Yagi Y, Nishida Y, Kataoka H, O'Connor MB, Mizoguchi A. 2009. A fat body-derived IGFlike peptide regulates postfeeding growth in Drosophila. Dev Cell 17: 885-891.

Okamoto N, Nishimori Y, Nishimura T. 2012. Conserved role for the Dachshund protein with Drosophila Pax6 homolog 
Eyeless in insulin expression. Proc Natl Acad Sci 109: 24062411.

Parks AL, Cook KR, Belvin M, Dompe NA, Fawcett R, Huppert K, Tan LR, Winter CG, Bogart KP, Deal JE, et al. 2004. Systematic generation of high-resolution deletion coverage of the Drosophila melanogaster genome. Nat Genet 36: 288-292.

Pierce SB, Costa M, Wisotzkey R, Devadhar S, Homburger SA Buchman AR, Ferguson KC, Heller J, Platt DM, Pasquinelli AA, et al. 2001. Regulation of DAF-2 receptor signaling by human insulin and ins-1, a member of the unusually large and diverse C. elegans insulin gene family. Genes Dev 15: 672-686.

Puig O, Marr MT, Ruhf ML, Tjian R. 2003. Control of cell number by Drosophila FOXO: Downstream and feedback regulation of the insulin receptor pathway. Genes Dev 17: 2006-2020.

Scott CD, Ballesteros M, Madrid J, Baxter RC. 1996. Soluble insulin-like growth factor-II/mannose 6-P receptor inhibits deoxyribonucleic acid synthesis in cultured rat hepatocytes. Endocrinology 137: 873-878.

Sharma Y, Cheung U, Larsen EW, Eberl DF. 2002. pPTGAL, a convenient Gal4 P-element vector for testing expression of enhancer fragments in Drosophila. Genesis 34: 115-118.

Slaidina M, Delanoue R, Grönke S, Partridge L, Léopold P. 2009. A Drosophila insulin-like peptide promotes growth during nonfeeding states. Dev Cell 17: 874-884.

Sloth Andersen A, Hertz Hansen P, Schaffer L, Kristensen C. 2000. A new secreted insect protein belonging to the immunoglobulin superfamily binds insulin and related peptides and inhibits their activities. I Biol Chem 275: 16948-16953.

Sousa-Nunes R, Yee LL, Gould AP. 2011. Fat cells reactivate quiescent neuroblasts via TOR and glial insulin relays in Drosophila. Nature 471: 508-512.

Thissen JP, Ketelslegers JM, Underwood LE. 1994. Nutritional regulation of the insulin-like growth factors. Endocr Rev 15: 80-101.

Valenzano KJ, Remmler J, Lobel P. 1995. Soluble insulin-like growth factor II/mannose 6-phosphate receptor carries multiple high molecular weight forms of insulin-like growth factor II in fetal bovine serum. I Biol Chem 270: 1644116448.

Veenstra JA, Agricola HJ, Sellami A. 2008. Regulatory peptides in fruit fly midgut. Cell Tissue Res 334: 499-516.

Vorlová S, Rocco G, Lefave CV, Jodelka FM, Hess K, Hastings ML, Henke E, Cartegni L. 2011. Induction of antagonistic soluble decoy receptor tyrosine kinases by intronic polyA activation. Mol Cell 43: 927-939.

Zaina S, Squire S. 1998. The soluble type 2 insulin-like growth factor (IGF-II) receptor reduces organ size by IGF-II-mediated and IGF-II-independent mechanisms. J Biol Chem 273: 28610 28616. 


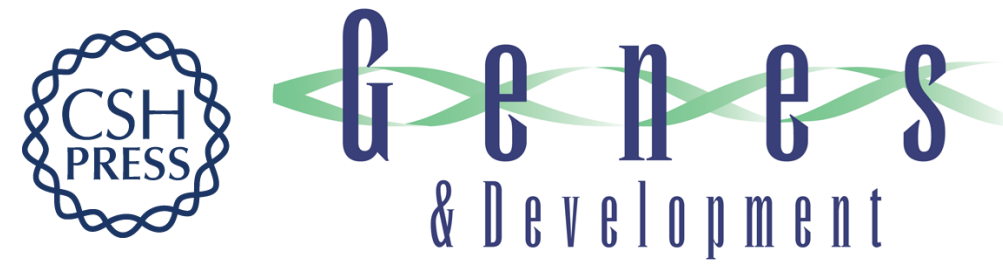

\section{A secreted decoy of InR antagonizes insulin/IGF signaling to restrict body growth in Drosophila}

Naoki Okamoto, Rinna Nakamori, Tomoka Murai, et al.

Genes Dev. 2013, 27:

Access the most recent version at doi:10.1101/gad.204479.112

Supplemental http://genesdev.cshlp.org/content/suppl/2013/01/10/27.1.87.DC1
Material

References This article cites 30 articles, 6 of which can be accessed free at: http://genesdev.cshlp.org/content/27/1/87.full.html\#ref-list-1

License

Email Alerting Receive free email alerts when new articles cite this article - sign up in the box at the top Service right corner of the article or click here.

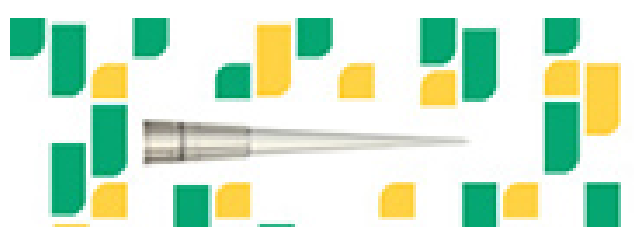

Focused on your science. 